\title{
Análisis del desarrollo histórico de la industria maquiladora de exportación en México: caso de Ciudad Juárez, Chihuahua.
}

Analysis of the historical development of the maquiladora export industry in Mexico: case of Ciudad Juárez, Chihuahua.

\section{Oscar Galván Mendoza ${ }^{1}$ y Jesús García Galaviz ${ }^{2}$}

\section{Resumen}

El objetivo de la presente investigación es el de analizar la evidencia científica que versa acerca del desarrollo de la industria maquiladora de exportación en México, particularmente en Ciudad Juárez. Al tener como antecedente la firma del acuerdo bracero agreement entre México y Estados Unidos de Norte América en el año de 1942, se realiza una aproximación teórica para comprender la evolución de la industria maquiladora de exportación específicamente en la región fronteriza de Ciudad Juárez. Adicionalmente, se presenta información de la situación actual de dicho sector.

Palabras clave: Industria maquiladora de exportación, Ciudad Juárez, evolución.

\section{Abstract}

The aim of the present investigation is of analyze the scientific evidence that deals with the development of maquiladora export industry in Mexico, particularly in Ciudad Juárez. Having as a precedent the signing of the bracero agreement between Mexico and the United States of North America in the year 1942, a theoretical approach is made to understand the evolution of the maquiladora export industry specifically in the border region of Ciudad Juarez. Additionally, information on the current situation of said sector is presented. Additionally, information on the current situation of said sector is presented.

Keywords: Maquiladora export industry, Ciudad Juarez, evolution.

\footnotetext{
1 Maestría en Administración por la Universidad Autónoma de Ciudad Juárez, Instituto de Ciencias Sociales y Administración. Av. Universidad y Heroico Colegio Militar Ciudad Juárez, Chih. Correo electrónico: noccarempo@gmail.com

2 PTC de Universidad Autónoma de Chihuahua, Facultad de ciencias políticas y sociales. Av. Henry Dunant \# 4612, Cd. Juárez Chihuahua. Correo electrónico:jesusgarciagalaviz@hotmail.com
} 


\section{Introducción}

La palabra maquiladora es un término derivado de makílah, una palabra de origen árabe, (Jiménez, 2008), que en sus primeros usos se relacionaba con la actividad de la molienda, haciendo referencia a la porción del grano, harina o aceite que se abona al molinero por sus servicios (Gómez, 2004). Con el paso de los años, el significado del término evolucionó hasta designar a (Douglas y Hansen, 2003) "cualquier actividad particular en un proceso industrial -por ejemplo, el ensamblaje o el embalaje- realizada por una parte que no es el fabricante original" (Angulo, 1990:139). Posteriormente, este término ha sido utilizado con el fin de identificar a aquellas industrias dedicadas a cualquier manufactura parcial, ensamble o empaque llevado a cabo por una empresa que no sea el fabricante original (Villalpando, 2004).

$\mathrm{Y}$ es que dado que la importancia de la industria maquiladora de exportación en la generación de empleo, exportaciones y divisas radica en su capacidad de evolución productiva, organizacional y tecnológica (Carrillo y García, 2002), se debe hacer mención que ésta es considerada la base del desarrollo industrial en el norte de México en función de que es uno de los núcleos más dinámicos de las exportaciones no tradicionales del país (Alonso, Carrillo y Contreras, 2002).

Sin embargo, aunque existen trabajos que versan sobre el desarrollo histórico y la importancia de la industria maquiladora de exportación en México haciendo mención de Ciudad Juárez como un referente de rápido crecimiento económico, una destacada infraestructura urbana e industrial y por su mano de obra especializada de bajo costo, son escasos los estudios que presenten un abordaje del desarrollo histórico de la industria maquiladora de exportación específicamente de Ciudad Juárez, Chihuahua. Con relación a esto, en el presente trabajo se plantea como objetivo general el analizar la evidencia científica que versa acerca del desarrollo de la industria maquiladora de exportación en México, particularmente en Ciudad Juárez, Chihuahua.

\section{Aspectos históricos de la industria maquiladora de exportación en el mundo}

Se presenta a continuación la Tabla 1 que muestra el surgimiento de la industria ensambladora a nivel mundial: 
pISSN: 2395-8758

eISSN: en trámite

Tabla 1: Expansión del procesamiento de artículos de exportación en el tercer mundo, 1960-1984 por país y fecha de establecimiento de las primeras instalaciones para el

\begin{tabular}{|c|c|c|c|c|c|}
\hline & $1960-1964$ & $1965-1969$ & $1970-1974$ & $1975-1979$ & $1980-1984$ \\
\hline Asia & Hong Kong & $\begin{array}{l}\text { Corea del sur } \\
\text { Taiwán Singapur } \\
\text { Filipinas India }\end{array}$ & Malasia & $\begin{array}{l}\text { Indonesia } \\
\text { Sri Lanka }\end{array}$ & $\begin{array}{l}\text { China } \\
\text { Tailandia } \\
\text { Bangladesh } \\
\text { Paquistán }\end{array}$ \\
\hline $\begin{array}{l}\text { Latinoamérica } \\
\text { y el caribe }\end{array}$ & Puerto Rico & $\begin{array}{l}\text { México } \\
\text { Rep. Dominicana } \\
\text { Panamá Brasil }\end{array}$ & $\begin{array}{l}\text { Haití } \\
\text { El Salvador } \\
\text { Guatemala } \\
\text { Colombia }\end{array}$ & $\begin{array}{l}\text { Jamaica } \\
\text { Honduras } \\
\text { Nicaragua } \\
\text { Chile }\end{array}$ & Costa Rica \\
\hline $\begin{array}{l}\text { África y } \\
\text { Medio }\end{array}$ & & Mauricio & $\begin{array}{l}\text { Túnez } \\
\text { Jordania }\end{array}$ & $\begin{array}{l}\text { Egipto } \\
\text { Siria } \\
\text { Liberia } \\
\text { Senegal }\end{array}$ & Chipre \\
\hline
\end{tabular}

Fuente: Wilson (1996:29).

Con base en el contenido de la Tabla 1, se destaca que el surgimiento de la industria ensambladora a nivel mundial se remonta a los años sesenta en Hong Kong y Puerto Rico, en el caso de México, éste se presenta en la década de los sesenta en México (Wilson, 1996).

\section{La industria maquiladora de exportación en México}

La industria maquiladora en México tiene como antecedente la firma del acuerdo bracero agreement entre México y Estados Unidos de Norte América en el año de 1942 (Villalpando, 2004). En función de contribuir a remediar la falta de mano de obra agrícola en Estados Unidos como consecuencia de la segunda guerra mundial (Albertí, 2012), dicho acuerdo consistía en modelo de migración pensado exclusivamente para hombres, que exigía un modelo de varón joven, soltero de origen rural, dedicado al medio rural, a quien se le proponía una contratación temporal (Durand y Arias, 2005). Tal programa facilitó el acceso de mano de obra de bajo costo a los productores norteamericanos, reclutando un total de cinco millones de trabajadores para laborar prioritariamente en el sector agrícola, en los ferrocarriles o en el sector minero (Albertí, 2012).

El origen de la maquila en México data en 1964, momento en que finalizó el Programa de Braceros y el retorno de 200000 trabajadores con el 


\section{DOXA}

pISSN: 2395-8758

eISSN: en trámite

consiguiente aumento del desempleo en los estados fronterizos (Gómez, 2004). En un intento de trasladar a esos estados la experiencia de las maquiladoras estadounidenses, el gobierno mexicano aprobó en 1965 el Programa de la Industria Maquiladora de Exportación -Programa de Industrialización Fronteriza, PIF- (Carrillo y Hualde, 1992). Este programa permitía importar desde Estados Unidos maquinaria, equipo y componentes libres de aranceles con la condición de que los últimos se procesaran y reexportaran hacia ese país y que los equipos se exportaran cuando ya no se necesitaran (Gómez, 2004). La finalidad del programa era atraer inversión extranjera, principalmente estadounidense, en una franja de 10 millas, donde se podían establecer ensambladoras o empresas que utilizaran insumos importados para su producción y ensamble, reexportando los bienes terminados a su planta matriz (Dutrénit y Vera, 2002). Considerada como una zona libre de comercio, dentro de esta franja se debían pagar los impuestos correspondientes al valor agregado generados por la mano de obra utilizada (Lowe y Kenney, 1999; Buitelaar, 2000); estas plantas ensambladoras fueron denominadas maquilas (Dutrénit y Vera, 2002).

Por otra parte, teniendo como objetivos el elevar el nivel de vida de sus habitantes, aumentar el consumo de la producción nacional en esas regiones, generar nuevos empleos, cambiar la apariencia física de las poblaciones fronterizas y elevar el nivel cultural (Jiménez, 2008), el programa maquilador se estableció como el primer esquema formal y explicito que adopto México para participar en la subcontratación internacional de servicios productivos (González y Barajas, 1989). Es a partir de la formalización del programa de industrialización fronteriza, lo que brinda la pauta para la división en etapas o periodos de desarrollo de la industria maquiladora de exportación (Jiménez, 2008). Dentro de la evidencia científica, se identificaron las siguientes etapas y sus respectivas características [ver Tabla 2]: 
pISSN: 2395-8758

eISSN: en trámite

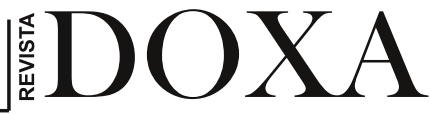

Tabla 2: Etapas o periodos de la IME en México

\begin{tabular}{|c|c|c|c|c|c|}
\hline \multicolumn{6}{|c|}{ FACTORES } \\
\hline & Crecimiento & $\begin{array}{l}\text { Auge y caidas } \\
\text { en el nivel de } \\
\text { actividad }\end{array}$ & $\begin{array}{l}\text { Economia, } \\
\text { producción }\end{array}$ & $\begin{array}{l}\text { Relaciones } \\
\text { laborales }\end{array}$ & $\begin{array}{c}\text { Generaciones y } \\
\text { fases }\end{array}$ \\
\hline $\begin{array}{l}\text { Primera } \\
\text { etapa o } \\
\text { periodo }\end{array}$ & $\begin{array}{l}1965-1980 \\
\text { Formación, } \\
\text { crecimiento y } \\
\text { estabilización de } \\
\text { la IME }\end{array}$ & $\begin{array}{c}\text { 1965-1974 } \\
\text { Instalación } \\
1974-1977 \\
\text { Primera } \\
\text { contracción } \\
1977-1980 \\
\text { Nuevo periodo } \\
\text { de crecimiento }\end{array}$ & $\begin{array}{c}1965-1982 \\
\text { Modelo de } \\
\text { integración } \\
\text { complementaria } \\
\text { de economías }\end{array}$ & $\begin{array}{c}1965-1982 \\
\text { Herencias } \\
\text { sindicales y } \\
\text { cambios } \\
\text { productivos } \\
\text { internacional } \\
\text { es }\end{array}$ & $\begin{array}{l}1965-1981 \\
\text { Primera } \\
\text { generación o } \\
\text { fase del } \\
\text { ensamble } \\
\text { ("maquila en } \\
\text { México") }\end{array}$ \\
\hline $\begin{array}{l}\text { Segunda } \\
\text { etapa o } \\
\text { periodo }\end{array}$ & $\begin{array}{c}1981-1990 \\
\text { Expansión y } \\
\text { nuevas } \\
\text { modalidades }\end{array}$ & $\begin{array}{c}\text { 1980-1983 } \\
\text { Segunda } \\
\text { contracción } \\
1983-1990 \\
\text { Recuperación } \\
\text { y } \\
\text { consolidación }\end{array}$ & $\begin{array}{c}\text { 1983-1994 } \\
\text { Reestructuración } \\
\text { productiva en } \\
\text { México }\end{array}$ & $\begin{array}{c}\text { 1983-1994 } \\
\text { Flexibilidad } \\
\text { sindical: de } \\
\text { la } \\
\text { negociación } \\
\text { a la colusión }\end{array}$ & $\begin{array}{l}\text { 1982-1994 } \\
\text { Segunda } \\
\text { generación o } \\
\text { fase de la } \\
\text { manufactura } \\
\text { ("hecho en } \\
\text { México") }\end{array}$ \\
\hline $\begin{array}{l}\text { Tercera } \\
\text { etapa o } \\
\text { periodo }\end{array}$ & $\begin{array}{l}1991-2000 \\
\text { Crecimiento } \\
\text { constante y el } \\
\text { TLCAN }\end{array}$ & $\begin{array}{l}1991-2000 \\
\text { Expansión de } \\
\text { la industria } \\
\text { electrónica y } \\
\text { autopartes }\end{array}$ & $\begin{array}{l}1995-2000 \\
\text { La entrada del } \\
\text { TLCAN }\end{array}$ & $\begin{array}{l}1995-2000 \\
\text { Apertura } \\
\text { comercial, } \\
\text { colaboración } \\
\text { y retroceso } \\
\text { social }\end{array}$ & $\begin{array}{l}\text { 1994- tercera } \\
\text { generación o } \\
\text { fase del diseño } \\
\text { ("creado en } \\
\text { México" }\end{array}$ \\
\hline $\begin{array}{l}\text { Cuarta } \\
\text { etapa o } \\
\text { periodo }\end{array}$ & $\begin{array}{c}\text { 2001-2004 } \\
\text { Desaceleración }\end{array}$ & $\begin{array}{l}\text { 2001-2004 } \\
\text { Contracción } \\
\text { por } \\
\text { desaceleración } \\
\text { económica } \\
\text { mundial }\end{array}$ & $\begin{array}{c}2001-2004 \\
\text { Desaceleración } \\
\text { económica y } \\
\text { perdida de } \\
\text { ventajas } \\
\text { competitivas }\end{array}$ & $\begin{array}{c}2001-2004 \\
\text { Nuevas } \\
\text { relaciones } \\
\text { laborales en } \\
\text { la } \\
\text { maquiladora }\end{array}$ & $\begin{array}{c}\text { Cuarta } \\
\text { generación o } \\
\text { fase de } \\
\text { coordinación } \\
\text { de múltiples } \\
\text { actividades con } \\
\text { base en } \\
\text { tecnologias de } \\
\text { la información }\end{array}$ \\
\hline
\end{tabular}

Fuente: Jiménez (2008:100).

Con base en el contenido de la Tabla anterior, es necesario mencionar que en el caso en particular de la primera etapa de desarrollo, la industria maquiladora de exportación fue considerada como un modelo de integración complementaria de economías o production sharing -el ciclo de vida del producto- (Carrillo, Hualde y Quintero, 2005), con la finalidad de atraer inversión extranjera (Dutrénit y Vera, 2002) y a la par, el generar empleos e integrar la tecnología a la industria nacional (Villalpando, 2004).

En lo que corresponde a la segunda etapa, Ludlow y de la Rosa (2008) le describen como un periodo de consolidación y expansión, ya que el proceso de ensamble ya no se concretaba solamente a la frontera norte sino que, 
además de seguir creciendo en esta región, se expandió al resto del país. Otra característica a considerar es la interacción entre las condicionantes laborales de los trabajadores y los requerimientos productivos internacionales (Quintero, 2006), "lo que para algunos sindicatos significó una moderación en su política de enfrentamiento y la búsqueda de acuerdos productivos y laborales consensuados, que se tradujo especialmente en un comportamiento más flexible" (p. 20). También se detectó que la actividad maquiladora presentó una leve caída provocada por una nueva política monetaria que contemplaba dos mecanismos básicos: el deslizamiento del peso y el control de cambios con estructura dual de precios: libre y controlado (Jiménez, 2008).

Por otro lado, la tercera etapa de desarrollo se caracteriza por la entrada del Tratado de Libre Comercio en América del Norte (TLCAN). Acorde a Gómez (2004), es a partir de este suceso cuando se produce el boom de la industria maquiladora, lo cual se refleja en el aumento en el número de establecimientos y en el empleo en dichas empresas. Por lo que México se ajustó rápidamente y comenzó a desmantelar su elevado nivel de proteccionismo en dos frentes: eliminando cuotas y permisos previos de importación, y bajando aranceles y reduciendo su dispersión (Ludlow y de la Rosa, 2008). Adicionalmente, dentro de dicha etapa se presenta "el establecimiento del primer centro de investigación y desarrollo de una empresa transnacional automotriz vinculado con la producción manufacturera de las maquiladoras en Ciudad Juárez" (Carrillo y Gomis, 2007:27). Dado el ambiente de competitividad en la economía externa, se hizo presente la difusión tecnológica, pues algunas industrias comienzan a trabajar con tecnología de punta, lo que se ha dado en llamar escalamiento industrial, presente en sectores como los de autopartes y electrónico (Ludlow y de la Rosa, 2008).

No obstante, en la cuarta etapa de desarrollo, la industria maquiladora de exportación mostró señales de agotamiento, evidenciando su vulnerabilidad a los factores externos a causa principalmente de la desaceleración de la economía norteamericana, principal destino de los productos maquiladores (Castilla y Torres, 2010). Es debido a la desaceleración económica estadounidense lo que generó un aumento considerable en el cierre de plantas y de despidos que se registraron a partir 
pISSN: 2395-8758

eISSN: en trámite

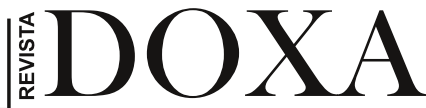

del año 2000 (de la O, 2006); así como también por la emergencia de nuevas zonas de maquiladoras -China, Centroamérica e India- con costos de producción menores a los encontrados en México (Castilla y Torres, 2010). Ese traslado de empresas hacia Centroamérica, fundamentalmente de la maquila tradicional (textil, vestido y alimentos) o de las secciones del proceso productivo de la maquila no tradicional (autopartes, electrónica y cómputo) a China, ocasiono una notable disminución de ventas (Ludlow y de la Rosa, 2008).

Basta con analizar la entrada de China en la Organización Mundial de Comercio a finales de 2001 y la influencia de la constante caída de aranceles en Estados Unidos (Rodríguez, 2003) para poder entender ese contexto de competencia internacional desfavorable para México, en donde los costos de operación por arriba de \$2.59 la hora, no podían competir con los costos de operación en China de menos de $\$ 1$ la hora (Jiménez, 2008). Por lo que el sector más afectado fue el de la industria electrónica, especialmente en la región fronteriza (GAO 2003). Ante esta situación, Contreras y Munguía (2007) detallan que de noviembre del 2000 a diciembre del 2001 se perdieron poco más de 250 mil puestos de trabajo; un año después, el empleo se había reducido un 20 por ciento y la producción un 30 por ciento.

Dicho lo anterior, Pérez (2006) enfatiza en que se debe recordar que la localización del país depende de los bajos salarios, como en los casos en donde la conexión entre la maquila y el aparato productivo del país huésped es poca. El mismo autor sostiene que otra fuente de problemas "que explican la crisis tienen que ver con el marco fiscal. Cabe recordar que uno de los factores de localización de las empresas era precisamente los incentivos fiscales de que gozó la industria maquiladora" (p. 235).

Sin embargo, cuando la economía de Estados Unidos comenzó a recuperarse, esta problemática económica se revirtió en parte, y al final del primer semestre de 2006 se tenían registradas un total de 2822 plantas y 1 223180 empleos (INEGI, 2007). La notable recuperación en los años posteriores indica que las maquiladoras siguieron teniendo un papel crucial en la economía mexicana, siendo una opción rentable para las transnacionales dedicadas a actividades altamente globalizadas (Contreras y Munguía, 2007). 
La industria maquiladora de exportación en Ciudad Juárez

Considerando que el gobierno mexicano siguió invirtiendo en la industrialización de la frontera después de la segunda guerra mundial, Lorey (1999) señala que esta región se rezagó con respecto a los principales centros del país -Monterrey, Guadalajara y Ciudad de México- en términos de desarrollo industrial. Por otro lado, en Ciudad Juárez, un grupo de ciudadanos distinguidos integrado por empresarios, líderes civiles, políticos, burócratas y representantes campesinos y sindicales, consideró que la ciudad que requería una dirección industrial nueva para el futuro (Douglas y Hansen, 2003). Los mismos autores detallan que hacia mediados de la década de los cincuenta, "se había puesto en marcha en esa ciudad fronteriza un movimiento encabezado por la Cámara Nacional de la Industria de la Transformación (Canacintra) para promover el establecimiento de industrias nacionales que permitieran sustituir las importaciones de bienes extranjeros" (p. 1047).

En los inicios de los años sesenta, el presidente Adolfo López Mateos le pidió a Antonio J. Bermúdez, el empresario más destacado de Ciudad Juárez, que encabezara una organización encargada de transformar la región de la frontera norte (Bermúdez, 1966). Por lo que el primer intento institucional de integración económica, política y cultural de esa zona fue la creación del Programa Nacional Fronterizo (Pronaf) de 1961 (Mungaray, 1998), teniendo como objetivo la creación de fuentes de trabajo para elevar la calidad de vida de la población, ampliando tanto la cobertura de servicios, como los niveles de cultura a fin de fortalecer la conciencia y la identidad nacionales (Mungaray y Moctezuma, 1984).

Cuatro años después, el gobierno mexicano aprobó el Programa de la Industria Maquiladora de Exportación -Programa de Industrialización Fronteriza, PIF- (Carrillo y Hualde, 1992), considerado como "un importante paliativo al intenso problema de desempleo en las ciudades fronterizas, pero también un modelo de industrialización ad hoc a las condiciones de apertura y desintegración que prevalecían en la frontera" (Mungaray, 1998:267).

Cabe señalar que en el año de 1966, la primera maquiladora que inició operaciones en Ciudad Juárez fue la empresa A.C. Nielsen de México, la cual elaboraba cupones comerciales (Gutiérrez, 2009). Un año después, se creó el parque industrial Antonio J. Bermúdez, el cual se destacó por un rápido crecimiento dado la amplia disponibilidad de infraestructura y 
pISSN: 2395-8758

eISSN: en trámite

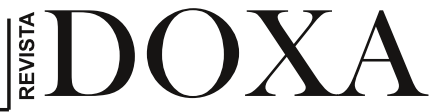

organización administrativa del mismo (Iglesias, 2014). Fue en ese parque industrial en donde se instaló en 1969 la empresa RCA Víctor de México S. A., planta de la rama electrónica que sería el prototipo moderno de las maquiladoras venideras (Gutiérrez, 2009).

A continuación se presenta la Figura 1, la cual contiene plasmados los sucesos históricos ya abordados con respecto al desarrollo de la industria maquiladora de exportación específicamente en Ciudad Juárez:

Figura 1: Desarrollo histórico de la industria maquiladora de exportación en Ciudad Juárez

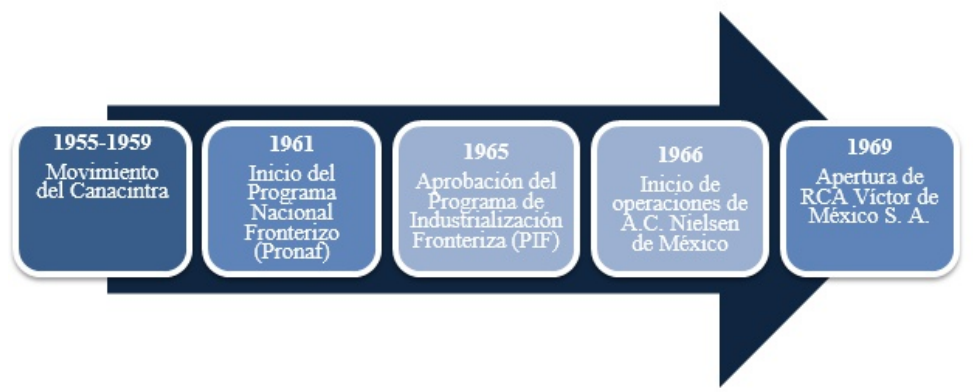

Fuente: Elaboración propia con base en Douglas y Hansen (2003) y Gutiérrez (2009).

Con el objetivo de brindar productos de buena calidad para poder retener al consumidor fronterizo dentro del comercio local, en 1970 la administración pública federal inició con el Programa de Comercialización Fronteriza (Mungaray, 1998). Por lo que en esa época Ciudad Juárez se posicionaba como el sitio preferido para los inversionistas dada su cercanía a El Paso, así como por su infraestructura urbana e industrial (Carrillo y Hernández, 1985). Posteriormente, en esa misma ciudad, el 7 de febrero de 1974 se creó la Asociación de Maquiladoras, la institución que representa en la actualidad a la industria maquiladora a través de acciones y servicios de calidad con eficacia y profesionalismo (INDEX, 2015). Otro suceso a considerar es la publicación del decreto para el apoyo a la Industria Manufacturera, Maquiladora y de Servicios de Exportación (IMMEX), el cual, según el Instituto Municipal de Investigación y Planeación (IMIP, 2014) data el $1^{\circ}$ de Noviembre de 2006 . Este programa brinda la posibilidad de importar de forma temporal y libre de impuestos aquellos bienes necesarios 


\section{DOXA}

pISSN: 2395-8758

eISSN: en trámite

para ser utilizados en un proceso industrial o de servicio destinado a la elaboración, transformación o reparación de mercancías, sea importación o exportación (Secretaría de Economía, 2006).

Situación actual de la industria maquiladora de exportación en el estado de Chihuahua

Acorde a datos del INDEX (2018), Ciudad Juárez es la principal ciudad que cuenta con el mayor número de empleados en la industria manufacturera, en Agosto de 2018 conto con 298, 262 trabajadores, representando casi el $66 \%$ de todo el estado. La misma asociación señala que la ciudad de Chihuahua es la segunda ciudad con mayores empleados contratados, de ahí le siguen Delicias, Cuauhtémoc, Parral y Casas Grandes [ver Gráfica 1].

En el estado se tienen registrados 454,034 empleados en el sector manufacturero.

Gráfica 1: Empleados Sector manufacturero en el estado de Chihuahua.

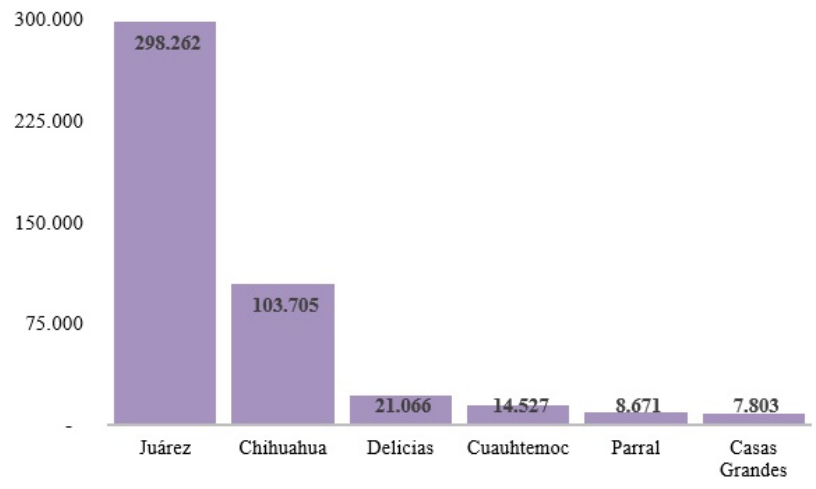

Fuente: Elaboración propia con base en INDEX (2018).

Durante el mes de Agosto de 2018 se tuvo un registro de 5,134 empresas operando bajo el esquema IMMEX en México; el estado de Baja California es el que tiene mayor número de empresas registradas seguido por Nuevo León. Los estados Baja California, Nuevo León, Chihuahua, Coahuila y Tamaulipas representan más del $55 \%$ el total del país de empresas con permiso IMMEX; en el caso en particular del estado de Chihuahua, éste 
pISSN: 2395-8758

eISSN: en trámite

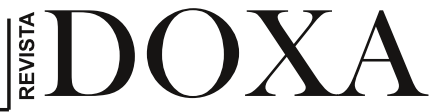

cuenta con 508 empresas [ver Gráfica 2].

Gráfica 2: Principales Estados con empresas IMMEX (Agosto 2018)

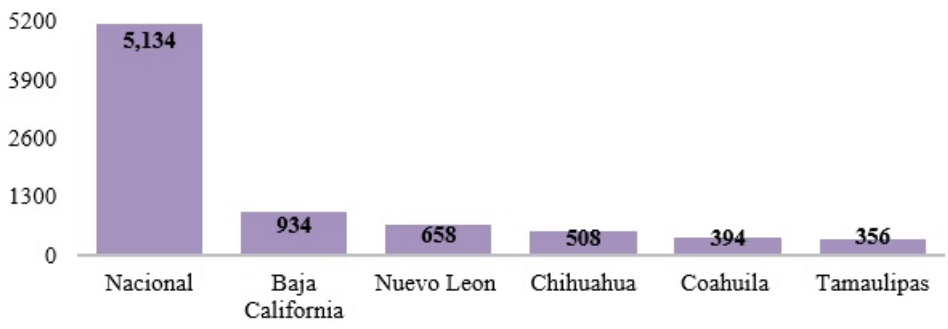

Fuente: INDEX (2018).

Específicamente en Ciudad Juárez, con base en los datos del INEGI (2018), en el mes de Julio del año 2018 se registró un total de 332 empresas activas operando bajo el esquema de la Industria Manufacturera, Maquiladora y de Servicios de Exportación (IMMEX) [ver Tabla 3]. Realizando una comparación con el mismo periodo del año anterior, se identifica que en el año 2018 se presentó un incremento considerable dado el establecimiento de 11 nuevas empresas.

Tabla 3: Industrias manufactureras activas en Ciudad Juárez (Enero 2017- Julio 2018)

\begin{tabular}{cccccc}
\hline Periodo & Empresas & Periodo & Empresas & Periodo & Empresas \\
\hline Enero/2017 & 322 & Agosto/2017 & 334 & Marzo/2018 & 332 \\
Febrero/2017 & 319 & Septiembre/2017 & 333 & Abril/2018 & 332 \\
Marzo/2017 & 321 & Octubre/2017 & 334 & Mayo/2018 & 332 \\
Abril/2017 & 321 & Noviembre/2017 & 334 & Junio/2018 & 332 \\
Mayo/2017 & 321 & Diciembre/2017 & 335 & Julio/2018 & 332 \\
Junio/2017 & 321 & Enero/2018 & 332 & & \\
Julio/2017 & 321 & Febrero/2018 & 332 & & \\
\hline
\end{tabular}

Fuente: Elaboración propia con base en INEGI (2018).

En esa misma línea de análisis, es necesario destacar que el año 2018 inició con el cierre de 3 empresas, ya que en Diciembre de 2017 se 
registraron un total de 335 empresas activas, y en el mes siguiente 332; de ahí su comportamiento ha sido estable con respecto al total de industrias manufactureras activas. En el caso en particular del año 2017, en el periodo correspondiente de Julio a Agosto se presentó un incremento de 13 nuevas industrias establecidas en Ciudad Juárez, éste ha sido un año de recuperación y generación de empleos en comparación con el año 2016 [ver Gráfica 3].

Gráfica 3: Industrias manufactureras activas en Ciudad Juárez (Enero 2016- Julio 2018)

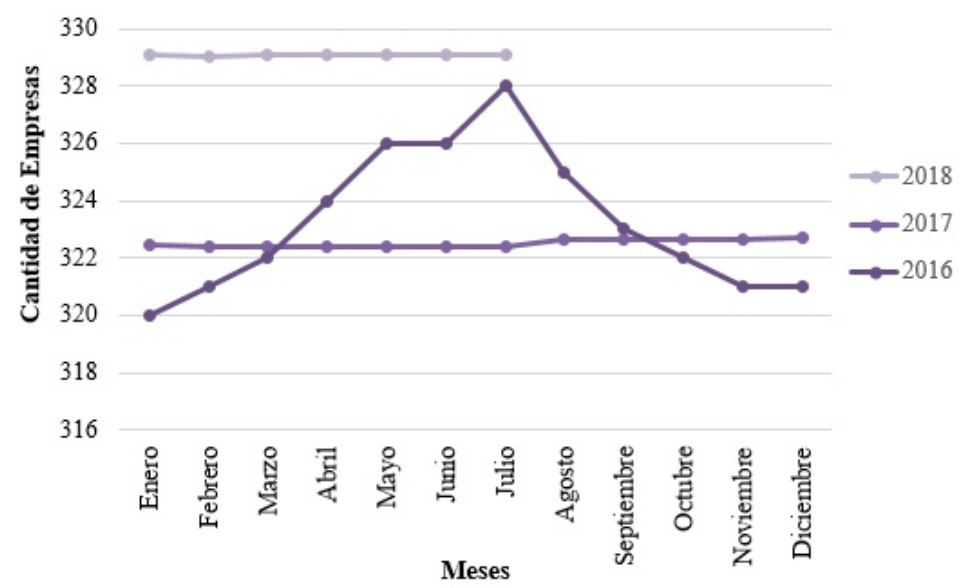

Fuente: Elaboración propia con base en INEGI (2018).

Por otra parte, teniendo consideración a las ciudades que son altamente generadoras de empleos dentro la industria manufacturera en México, Ciudad Juárez ocupa el primer lugar al representar el 27\% del total nacional, seguida por la ciudad de Tijuana. Se presenta a continuación la Gráfica 4, la cual muestra el contenido del cual se hace referencia:

Gráfica 4: Principales Ciudades Empleadoras (Agosto 2018)

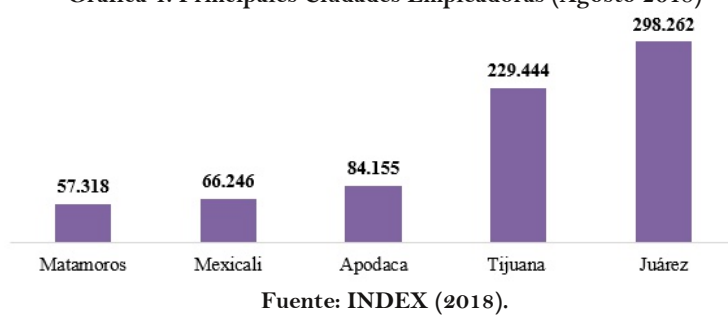


Finalmente, se muestra a continuación la Gráfica 5, la cual contiene un análisis de la contratación de empleados del sector manufacturero en Ciudad Juárez correspondiente al periodo agosto 2017- agosto 2018:

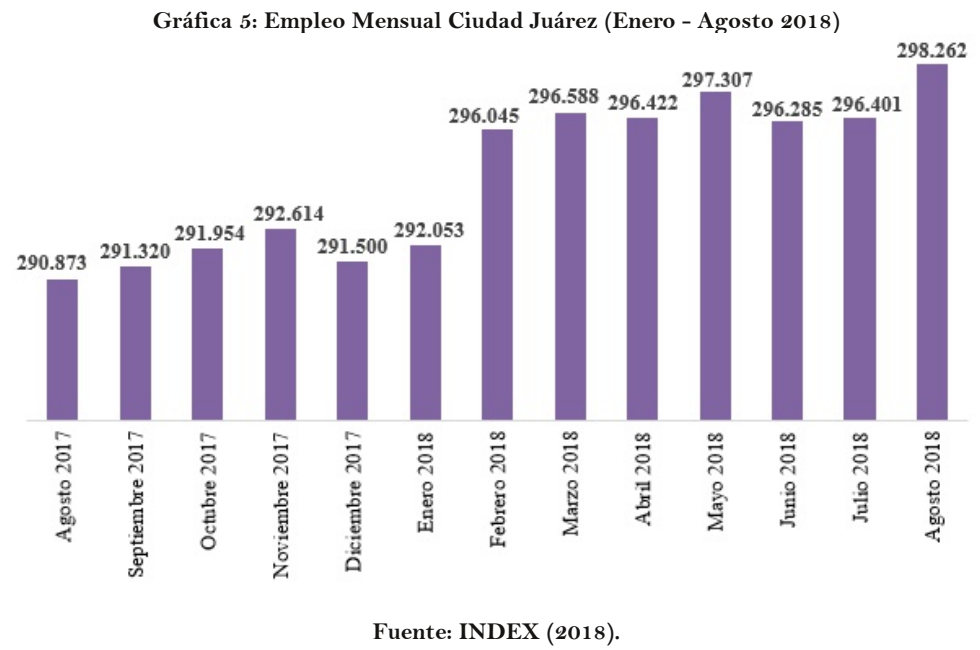

Con base en el contenido del grafico anterior, se puede afirmar que en Ciudad Juárez durante el periodo de enero a agosto de 2018 se presentó la creación de 6,209 nuevos empleos. Acorde al INDEX (2018), de un total de 298,262 empleados, el 74\% corresponde a la categoría de empleados directos (operadores y técnicos), el $17 \%$ pertenece a la categoría de empleados indirectos (personal de producción que no participa directamente en la transformación de la materia prima, como el gerente de producción, supervisor, superintendente), el $1 \%$ corresponde a la categoría staff (solamente gerentes) y el $8 \%$ restante atañe a los empleados de la categoría administrativa (personal de oficina). Quedando las equivalencias de la siguiente forma:

*Empleados directos: 220,714

*Empleados staff: 2,983

*Empleados indirectos: 50,704

*Empleados administrativos: 23,861 


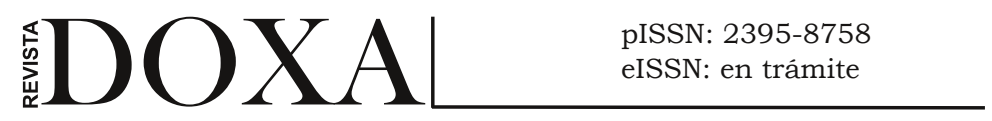

*Total de empleados: 298,262.

\section{Conclusiones}

Esta investigación se vuelve relevante al identificar que hay muy pocos estudios en México que abordan la evolución de la industria maquiladora de exportación en Ciudad Juárez. Teniendo como antecedente el acuerdo bracero agreement entre México y Estados Unidos de Norte América en el año de 1942, el origen de la maquila en México data en 1964, momento en que finalizó dicho acuerdo, presentándose un aumento considerable del desempleo en los estados fronterizos.

En el caso de Ciudad Juárez, fue hasta los inicios de los años sesenta cuando Antonio J. Bermúdez encabezo una organización que se encargó de transformar la región de la frontera norte con la creación del Programa Nacional Fronterizo (Pronaf) de 1961, teniendo como objetivo la creación de fuentes de trabajo para elevar la calidad de vida de la población. Cuatro años después, el gobierno mexicano aprobó el Programa de la Industria Maquiladora de Exportación -Programa de Industrialización Fronteriza, PIF- ante el intenso problema de desempleo en las ciudades fronterizas.

Cabe señalar que, si bien es cierto que en el año de 1966 la primera maquiladora que inició operaciones en Ciudad Juárez fue la empresa A.C. Nielsen de México, también se detectó la existencia de otras seis empresas que ya se encontraban establecidas y operaban de la misma manera que una maquiladora antes de 1966, pero no se reconocían como tal debido al desconocimiento del término, por lo que se integraron en ese mismo año al Programa de Industrialización Fronteriza.

Posteriormente, en 1967 se creó el parque industrial Antonio J. Bermúdez, en donde, dos años después se instaló la empresa RCA Víctor de México S. A., planta de la rama electrónica que sería el prototipo moderno de las maquiladoras venideras. Es aquí donde se debe resaltar que en diversos estudios se señala a dicha empresa como la primera industria maquiladora establecida en Ciudad Juárez, Chihuahua, esta situación debe ser tomada en cuenta ya que se brinda un abordaje erróneo al comprender la historia de la industria maquiladora de exportación en Ciudad Juárez, considerando que se tiene el registro de la empresa A.C. Nielsen de México tres años antes. 
pISSN: 2395-8758

eISSN: en trámite

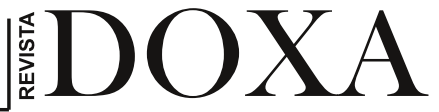

Con respecto a la situación actual de Ciudad Juárez, Chihuahua, con base en la información ya presentada, se afirma que Ciudad Juárez es la principal ciudad que cuenta con el mayor número de empleados en la industria manufacturera, ya que son 298,262 personas las que son empleadas por esta industria, lo que representa casi el $66 \%$ de todo el estado (hasta Agosto de 2018), éstas están distribuidas en un total de 332 empresas activas operando bajo el esquema de la Industria Manufacturera, Maquiladora y de Servicios de Exportación (IMMEX). De ahí que se le catalogue como la ciudad altamente generadora de empleo.

Bajo ese panorama, se aprecia la enorme capacidad de adaptación de Ciudad Juárez ante numerosos e importantes cambios dentro del entorno y la estructura de la fuerza del trabajo, en donde la globalización y la inestabilidad de la economía influyen diariamente en la conducta de las organizaciones.

\section{Referencias}

Albertí, A. (2012). Una reflexión teórica en torno a las migraciones laborales, a través del caso histórico del programa braceros (19421964). Nómadas. Critical Journal of Social and Juridical Sciences.

Alonso, J., Carrillo, J. y Contreras, Ó. (2002). Aprendizaje tecnológico en las maquiladoras del norte de México. Frontera norte, 14(27), 43-82.

Angulo, C. (1990). Foreign Investment and the Maquiladora Export Industry. Inversion Extranjera Directa-Direct Foreign Investment. Mexico, D.F.: Banamex, 139-143.

Bermúdez, A. (1966). El rescate del mercado fronterizo. Eufesa: México.

Buitelaar, R. (2000). Maquila, Economic Reform and Corporate Strategies. World Development 28(9): 1627-1642

Carrillo, J. y García, H. (2002). Evolución de las maquiladoras y el rol del gobierno y del mercado en la seguridad en el trabajo. Papeles de población, 8(33), 173-198.

Carrillo, J. y Gomis, R. (2003). Los retos de las maquiladoras ante la pérdida de competitividad. Comercio Exterior, 53 (4), 318-327.

Carrillo J. y Hernández, A. (1985). Mujeres fronterizas en la industria maquiladora. Secretaria de Educación Pública y Centro de Estudios Fronterizos: México, D.F. 
Carrillo, J. y Hualde, A. (1992). Empresas, maquiladoras y Tratado trilateral de Libre Comercio. Empleo, eslabonamiento y expectativas. Colección Cuadernos. El Colegio de la Frontera Norte.

Carrillo, J., Hualde, A. y Quintero, C. (2005). Recorrido por la historia de las maquiladoras en México, Comercio Exterior: Revista de Análisis Económico, 55 (1), 30-42.

Castilla, B. y Torres, B. (2010). Crisis sobre crisis en la industria maquiladora de exportación y sus consecuencias en la ocupación. El caso de Yucatán. El Cotidiano, (162), 43-56.

Contreras, Ó. y Munguía, L. (2007). Evolución de las maquiladoras en México: política industrial y aprendizaje tecnológico. Región y Sociedad, 19 (spe), 71-87.

de la O, M. (2006). Geografía del trabajo femenino en las maquiladoras de México. Papeles de población, 12 (49), 91-126.

Douglas, L. y Hansen, L. (2003). The origins of the Maquila industry in Mexico. Comercio exterior, 53(11), 1-16.

Durand, J. y Arias, P. (2005). La vida en el norte: Historia e iconografía de la migración México-Estados Unidos. Guadalajara, Jalisco: Universidad de Guadalajara.

Dutrénit, G. y Vera, A. (2002). Rompiendo paradigmas: acumulación de capacidades tecnológicas en la maquila de exportación. Innovación \& Competitividad, 2(6), 11-15.

General Accounting Office-GAO (2003). Mexico's Maquiladora Decline Affects U.S.-Mexico Border Communities and Trade; Recovery Depends in Part on Mexico's Actions. Documento. Washington.

Gómez, C. (2004). El desarrollo de la industria de la maquila en México, Problemas del Desarrollo 35 (138), 57-83.

González, B. y Barajas, R. (1989). Las maquiladoras: ajuste estructural y desarrollo regional. Tijuana: El Colegio de la Frontera Norte.

Gutiérrez, L. (2009). Ciudad Juárez en los sesenta: la estructura urbana en transición. Nóesis. Revista de Ciencias Sociales y Humanidades, 18 (36), 128-154.

Iglesias, D. (2014). Incidencia De Las Políticas Públicas En El Crecimiento De Los Parques Industriales En México, 1953-2010. Regional and Sectoral Economic Studies, 14(1), 21 1-222. 
IMIP. (2014). Directorio georreferenciado de parques, zonas industriales e industrias en Ciudad Juárez. Actualización diagnóstica sociodemográfica y económica del programa de desarrollo urbano de Ciudad Juárez, Chih. Recuperado de: http://www.imip.org.mx/directorio/catalogo.pdf

INDEX. (2015). Sobre AMAC-Index Juárez. Recuperado de: https://indexjuarez.com/nosotros

INDEX. (2018). Información general IMMEX. Información Estadística Mensual. Recuperado de: https://indexjuarez.com/wpcontent/uploads/2018/10/OCTUBRE-5.pdf

INEGI. (2007). Industria maquiladora de exportación. Estadísticas económicas. Recuperado de: http://internet.contenidos.inegi.org.mx/contenidos/Productos/pr od_serv/contenidos/espanol/bvinegi/productos/continuas/econo micas/maquiladora/ime/ime.pdf

INEGI. (2018). Banco de Información Económica. Recuperado de: http://www.inegi.org.mx/sistemas/bie/

Jiménez, P. (2008). Socialización Organizacional en la Industria Maquiladora Fronteriza del Norte de México: Casos en Ciudad Juárez (Tesis doctoral) Doctorado en Ciencias de la Administración, Universidad Nacional Autónoma de México, México, Distrito Federal.

Lorey, D. (1999). The US-Mexican Border in the Twentieth Century: A History of Economic and Social Transformation. Rowman \& Littlefield: Mary Land.

Lowe, N. y Kenney, M. (1999). Foreign Investment and the Global Geography of Production: Why the Mexican Consumer Electronics Industry Failed. World Development 27(8): 14271443.

Ludlow, J. y de la Rosa, J. (2009). Las exportaciones de maquila en México y el vínculo con las importaciones de EUA. Análisis Económico, XXIV (55), 155-177.

Mungaray, A. (1998). Maquiladoras y organización industrial en la frontera norte de México. Comercio exterior, 48(4), 266-272.

Mungaray, A. y Moctezuma, P. (1984). La disputa del mercado fronterizo 1960-1983. Estudios Fronterizos, 1, 89-111. 
Pérez, C. (2006). Crisis y recuperación de la Industria Maquiladora de Exportación, 2000-2004. Análisis Económico, XXI (48), 229-256.

Quintero, C. (2006). El sindicalismo en las maquiladoras: La persistencia de lo local en la globalización. Desacatos, (21), 11-28.

Rodríguez, M. (2003). Ingreso de china a la organización mundial de comercio. su primer impacto sobre el comercio mundial. Problemas del Desarrollo. Revista Latinoamericana de Economía, 34 (134), 4973.

Secretaría de Economía. (2006). Industria manufacturera, maquiladora y de servicio de exportación. Instrumentos de comercio exterior. Recuperado de: http://www.20062012.economia.gob.mx/comunidad-negocios/industria-ycomercio/instrumentos-de-comercio-exterior/immex

Villalpando, P. (2004). La evolución de la industria maquiladora en México (The evolution of maquiladora industry in México). Innovaciones de negocios, 1(2), 321-330.

Wilson, P. (1996). Las nuevas maquiladoras de México. Exportaciones y desarrollo local. México: Universidad de Guadalajara. 